\title{
Lower Bounds for the Blow up Time to a Coupled Nonlinear Hyperbolic Type Equations
}

\author{
Erhan Pişkin ${ }^{1 *}$, Yavuz Dinç² ${ }^{2}$ Cemil Tunç ${ }^{3}$
}

\section{Abstract}

The initial and Dirichlet boundary value problem of nonlinear hyperbolic type equations in a bounded domain is studied. We established a lower bounds for the blow up time.

Keywords: Hyperbolic type equations, Lower bounds, Nonlinear damping term.

2010 AMS: Primary 35B44, Secondary 35L56

${ }^{1}$ Dicle University, Department of Mathematics, Diyarbakır, Turkey, ORCID: 0000-0001-6587-4479

${ }^{2}$ Mardin Artuklu University, Department of Electronics Technical Vocational School of Higher Education, Mardin, Turkey, ORCID: 0000-0003-0897-4101

${ }^{3}$ Van Yuzuncu Yil University, Department of Mathematics, Van, Turkey, ORCID: 0000-0003-2909-8753

*Corresponding author: episkin@dicle.edu.tr

Received: 24 January 2020, Accepted: 24 February 2020, Available online: 25 March 2020

\section{Introduction}

In this paper, we deal with the lower bounds of the blow up time of solutions of the following hyperbolic type equations

$$
\left\{\begin{array}{ccc}
u_{t t}-\operatorname{div}\left(\rho\left(|\nabla u|^{2}\right) \nabla u\right)-\Delta u_{t t}+\left|u_{t}\right|^{m-1} u_{t}=f_{1}(u, v), & (x, t) \in \Omega \times(0, T), \\
v_{t t}-\operatorname{div}\left(\rho\left(|\nabla v|^{2}\right) \nabla v\right)-\Delta v_{t t}+\left|v_{t}\right|^{r-1} v_{t}=f_{2}(u, v), & (x, t) \in \Omega \times(0, T), \\
u(x, 0)=u_{0}(x), & u_{t}(x, 0)=u_{1}(x), & x \in \Omega, \\
v(x, 0)=v_{0}(x), & v_{t}(x, 0)=v_{1}(x), & x \in \Omega, \\
u(x, t)=v(x, t)=0, & x \in \partial \Omega,
\end{array}\right.
$$

where $\Omega \subset R^{n}(n=1,2,3)$ is a bounded domain with a sufficiently smooth boundary $\partial \Omega ; m, r \geq 1$ are constants, and $f_{i}(u, v): R^{2} \rightarrow R(i=1,2)$ are functions which will be specified later. Also,

$$
\rho(s)=b_{1}+b_{2} s^{q}, \quad q, b_{1}, b_{2} \geq 0 .
$$

In the absence of the dispersion terms $\left(\Delta u_{t t}\right.$ and $\left.\Delta v_{t t}\right)$, eq. (1.1) reduces to the following system

$$
\left\{\begin{array}{c}
u_{t t}-\operatorname{div}\left(\rho\left(|\nabla u|^{2}\right) \nabla u\right)-\Delta u_{t t}+\left|u_{t}\right|^{m-1} u_{t}=f_{1}(u, v), \\
v_{t t}-\operatorname{div}\left(\rho\left(|\nabla v|^{2}\right) \nabla v\right)-\Delta v_{t t}+\left|v_{t}\right|^{r-1} v_{t}=f_{2}(u, v) .
\end{array}\right.
$$

In [1], Wu et al. considered the global existence and the blow up of the solution of the problem (1.2). Later, Fei and Hongjun [2] improved the blow up result in [1]. Finally, in [3], Pişkin and Polat studied the existence, the decay and the blow up of the solutions for the problem (1.2). 
The aim of this paper note is to derive a lower bound for the blow up time occurs. Before stating our main theorem, we give some notations, lemmas and theorems.

\section{Preliminaries}

In this paper, we denote $\|\cdot\|=\|\cdot\|_{L^{2}(\Omega)}$ and $\|\cdot\|_{p}=\|\cdot\|_{L^{p}(\Omega)}$. Moreover, $c_{i}(i=1,2, \ldots)$ are arbitrary constants.

Let

$$
f_{1}(u, v)=(p+1)\left[a|u+v|^{p-1}(u+v)+b|u v|^{\frac{p-1}{2}} v\right]
$$

and

$$
f_{2}(u, v)=(p+1)\left[a|u+v|^{p-1}(u+v)+b|u v|^{\frac{p-1}{2}} u\right],
$$

where $a, b>0$ are constant and $p$ satisfies

$$
\left\{\begin{array}{c}
1<p \text { if } n \leq 2 \\
1<p \leq \frac{n}{n-2} \quad \text { if } n>2
\end{array}\right.
$$

By a simple calculation, we have

$$
u f_{1}(u, v)+v f_{2}(u, v)=(p+1) F(u, v), \quad(u, v) \in R^{2}
$$

where

$$
F(u, v)=\left[a|u+v|^{p+1}+2 b|u v|^{\frac{p+1}{2}}\right] .
$$

We define

$$
J(t)=\frac{1}{2}\left[b_{1}\left(\|\nabla u\|^{2}+\|\nabla v\|^{2}\right)\right]+\frac{1}{2 q+2}\left[b_{2}\left(\|\nabla u\|_{2 q+2}^{2 q+2}+\|\nabla v\|_{2 q+2}^{2 q+2}\right)\right]-\int_{\Omega} F(u, v) d x
$$

and

$$
I(t)=\left[b_{1}\left(\|\nabla u\|^{2}+\|\nabla v\|^{2}\right)\right]+\left[b_{2}\left(\|\nabla u\|_{2 q+2}^{2 q+2}+\|\nabla v\|_{2 q+2}^{2 q+2}\right)\right]-(p+1) \int_{\Omega} F(u, v) d x
$$

We also define the energy functional as follows

$$
\begin{aligned}
E(t)= & \frac{1}{2}\left(\left\|u_{t}\right\|^{2}+\left\|v_{t}\right\|^{2}\right)+\frac{1}{2}\left[b_{1}\left(\|\nabla u\|^{2}+\|\nabla v\|^{2}\right)\right] \\
& +\frac{1}{2 q+2}\left[b_{2}\left(\|\nabla u\|_{2 q+2}^{2 q+2}+\|\nabla v\|_{2 q+2}^{2 q+2}\right)\right]+\frac{1}{2}\left(\left\|\nabla u_{t}\right\|^{2}+\left\|\nabla v_{t}\right\|^{2}\right)-\int_{\Omega} F(u, v) d x .
\end{aligned}
$$

We also define

$$
W_{-}=\left\{(u, v):(u, v) \in W_{0}^{1,2 q+2}(\Omega) \times W_{0}^{1,2 q+2}(\Omega), I(u, v)<0\right\} .
$$

The next lemma shows that our energy functional (2.6) is a nonincreasing function along the solution of (1.1).

Lemma 2.1. Energy functional is a nonincreasing function for $t \geq 0$ and

$$
E^{\prime}(t)=-\left(\left\|u_{t}\right\|_{m+1}^{m+1}+\left\|v_{t}\right\|_{r+1}^{r+1}\right) \leq 0
$$

Proof. Multiplying the first equation in (1.1) by $u_{t}$ and the second equation by $v_{t}$, integrating over $\Omega$. Then integrating by parts, we get

$$
E(t)-E(0)=-\int_{0}^{t}\left(\left\|u_{\tau}\right\|_{m+1}^{m+1}+\left\|v_{\tau}\right\|_{r+1}^{r+1}\right) d \tau \text { for } t \geq 0
$$


Lemma 2.2. (Sobolev-Poincare inequality) [4]. Let

$$
\left\{\begin{array}{l}
2 \leq p<\infty ; \quad n=1,2 \\
2 \leq p \leq \frac{2 n}{n-2} ; \quad n \geq 3
\end{array}\right.
$$

then there is a constant $C_{*}=C_{*}(\Omega, p)$ such that

$$
\|u\|_{p} \leq C_{*}\|\nabla u\|, \quad \forall u \in H_{0}^{1}(\Omega) .
$$

Lemma 2.3. [5, 6] There exist two positive constants $c_{1}$ and $c_{2}$ such that

$$
\int_{\Omega}\left|f_{1}(u, v)\right|^{2} d x \leq c_{1}\left(\|\nabla u\|^{2}+\|\nabla v\|^{2}\right)^{p}
$$

and

$$
\int_{\Omega}\left|f_{2}(u, v)\right|^{2} d x \leq c_{2}\left(\|\nabla u\|^{2}+\|\nabla v\|^{2}\right)^{p}
$$

are satisfied.

The local existence theorem which can be established combining the arguments of [3].

Theorem 2.4. (Existence-uniqueness). Assume that (2.1) holds. Then further that $u_{0}, v_{0} \in W_{0}^{1,2 q+2}(\Omega) \cap L^{p+1}(\Omega)$ and $u_{1}, v_{1} \in L^{2}(\Omega)$.Then the system (1.1) has a unique local solution

$$
u, v \in C\left([0, T) ; W_{0}^{1,2 q+2}(\Omega) \cap L^{p+1}(\Omega)\right) .
$$

Theorem 2.5. [7]. Suppose that $r>\max \{p, q\}$ and $E(0)<0$ hold. Then the solution $u$ of the system blows up in finite time $T^{*}$.

\section{Lower bound for blow up time}

In this section, our aim is to determine a lower bound for blow up time of the system (1.1).

Theorem 3.1. Let $u_{0}, v_{0} \in W_{0}^{1,2 q+2}(\Omega) \cap L^{p+1}(\Omega), u_{1}, v_{1} \in L^{2}(\Omega),\left(u_{0}, v_{0}\right) \in W_{-}$, and $1<p, q<r$. Assume that (2.1) holds. Then the solutions $u$ of the problem (1.1) become unbounded at finite time $T^{*}$. Also, the lower bounds for the blow up time is given by

$$
\int_{\psi(0)}^{\infty} \frac{d \psi(z)}{\psi(\tau)+E(0)+\frac{c_{1}+c_{2}}{2}(p+1)^{p} \psi^{p}(\tau)} \leq T^{*} .
$$

Proof. We define

$$
\psi(t)=\int_{\Omega} F(u, v) d x
$$

By taking a derivative of (3.1), we get

$$
\psi^{\prime}(t)=\int_{\Omega}\left(u_{t} F_{u}+v_{t} F_{v}\right) d x
$$

Thanks to Young's inequality, we have

$$
\psi^{\prime}(t) \leq \frac{1}{2} \int_{\Omega}\left(u_{t}^{2}+v_{t}^{2}\right) d x+\frac{1}{2} \int_{\Omega}\left(F_{u}^{2}+F_{v}^{2}\right) d x .
$$

By the Lemma 3, we get

$$
\psi^{\prime}(t) \leq \frac{1}{2} \int_{\Omega}\left(u_{t}^{2}+v_{t}^{2}\right) d x+\frac{c_{1}+c_{2}}{2}\left(\|\nabla u\|^{2}+\|\nabla v\|^{2}\right)^{p}
$$


Since $I(t)<0$, we have

$$
b_{1}\left(\|\nabla u\|^{2}+\|\nabla v\|^{2}\right)+b_{2}\left(\|\nabla u\|_{2 q+2}^{2 q+2}+\|\nabla v\|_{2 q+2}^{2 q+2}\right) \leq(p+1) \int_{\Omega} F(u, v) d x .
$$

Inserting (3.4) into (3.3), we have

$$
\begin{aligned}
\psi^{\prime}(t) & \leq \frac{1}{2} \int_{\Omega}\left(u_{t}^{2}+v_{t}^{2}\right) d x+\frac{c_{1}+c_{2}}{2}\left((p+1) \int_{\Omega} F(u, v) d x\right)^{p} \\
& =\frac{1}{2} \int_{\Omega}\left(u_{t}^{2}+v_{t}^{2}\right) d x+\frac{c_{1}+c_{2}}{2}(p+1)^{p}\left(\int_{\Omega} F(u, v) d x\right)^{p} \\
& =\frac{1}{2} \int_{\Omega}\left(u_{t}^{2}+v_{t}^{2}\right) d x+\frac{c_{1}+c_{2}}{2}(p+1)^{p} \psi^{p}(t)
\end{aligned}
$$

By the definition $E(t)$, we get

$$
\begin{aligned}
& (q+1)\left(\left\|u_{t}\right\|^{2}+\left\|v_{t}\right\|^{2}\right)+(q+1) b_{1}\left(\|\nabla u\|^{2}+\|\nabla v\|^{2}\right) \\
& +b_{2}\left(\|\nabla u\|_{2 q+2}^{2 q+2}+\|\nabla v\|_{2 q+2}^{2 q+2}\right)+(q+1)\left(\left\|\nabla u_{t}\right\|^{2}+\left\|\nabla v_{t}\right\|^{2}\right) \\
= & (2 q+2) E(t)+(2 q+2) \int_{\Omega} F(u, v) d x \\
\leq & (2 q+2) E(0)+(2 q+2) \psi(t)
\end{aligned}
$$

Combining (3.5) and (3.6), we have

$$
\psi^{\prime}(t) \leq \psi(t)+E(0)+\frac{c_{1}+c_{2}}{2}(p+1)^{p} \psi^{p}(t)
$$

Applying Theorem 5, we have

$$
\lim _{t \rightarrow T^{*}} \int_{\Omega} F(u, v) d x=\infty
$$

According to (3.7), (3.8), we have

$$
\int_{\psi(0)}^{\infty} \frac{d \psi(z)}{\psi(\tau)+E(0)+\frac{c_{1}+c_{2}}{2}(p+1)^{p} \psi^{p}(\tau)} \leq T^{*}
$$

This completes the proof.

\section{References}

[1] J. Wu, S. Li, S. Chai, Existence and nonexistence of a global solution for coupled nonlinear wave equations with damping and source, Nonlinear Anal., 72(11) (2010), 3969-3975.

[2] L. Fei, G. Hongjun, Global nonexistence of positive initial-energy solutions for coupled nonlinear wave equations with damping and source terms, Abstr. Appl. Anal., (2011) 1-14.

[3] E. Pişkin, N. Polat, Global existence, decay and blowup solution for coupled nonlinearwave equations with damping and source terms, Turk. J. Math., 37 (2013), 633-651.

[4] R.A. Adams, J.J.F. Fournier, Sobolev Spaces, Academic Press, 2003.

[5] A. Peyravi, Lower bounds of blow up time for a system of semilinear hyperbolic Petrovsky equations, Acta Math. Sci. 36B(3) (2016), 683-688.

[6] E. Pişkin, Lower bounds for blow up time of coupled nonlinear Klein-Gordon equations, Gulf Journal of Mathematics, 5(2) (2017), 56-61.

[7] N. Mezaour, E. Pişkin, Decay rate and blow up solutions for coupled quasilinear system, Boletin de la Sociedad Matematica Mexicana. (in press) 